\title{
HISTORIA
}

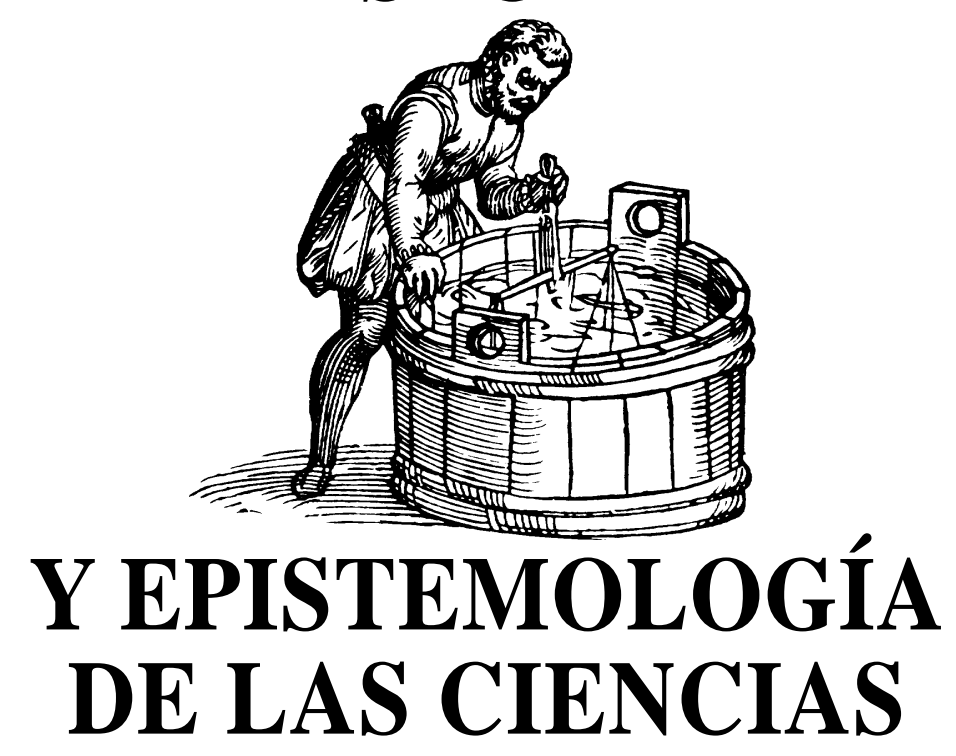

\section{UNA PROPUESTA PARA ESTRUCTURAR LA ENSEÑANZA DE LA FILOSOFÍA DE LA CIENCIA PARA EL PROFESORADO DE CIENCIAS EN FORMACIÓN}

\author{
ADÚRIZ-BRAVO, AGUSTÍN ${ }^{1}$, IZQUIERDO, MERCÈ ${ }^{1}$ y ESTANY, ANNA ${ }^{2}$ \\ ${ }^{1}$ Departament de Didàctica de les Matemàtiques i de les Ciències Experimentals \\ ${ }^{2}$ Departament de Filosofia \\ Universitat Autònoma de Barcelona \\ Edifici G5. Facultat de Ciències de l'Educació. Campus UAB \\ 08193 Bellaterra. Barcelona \\ agustin.aduriz@campus.uab.es
}

\begin{abstract}
Resumen. Desde la didáctica de las ciencias se considera que el conocimiento acerca de la naturaleza de la ciencia es uno de los componentes fundamentales de la alfabetización científica general. Esto lleva a la necesidad de introducir la filosofía de la ciencia en la formación inicial y continuada del profesorado de ciencias. En este trabajo se presenta un marco conceptual que puede dar lugar a la formulación de criterios teóricos de selección de los contenidos de la filosofía de la ciencia. Se utiliza este marco para analizar varias propuestas de formación metateórica del profesorado de ciencias.

Palabras clave. Filosofía de la ciencia, aportaciones a la didáctica de las ciencias, formación del profesorado, campos teóricos estructurantes, racionalidad y realismo.
\end{abstract}

Summary. It is acknowledged within didactics of science that knowledge about the nature of science is one of the fundamental components of general scientific literacy. This fact generates the need to introduce the philosophy of science in pre- and in-service science teacher education. In this paper, we present a conceptual framework that may result in theoretical criteria to select contents from the philosophy of science. This framework is used to analyse several proposals to teach the philosophy of science to science teachers.

Keywords. Philosophy of science, contributions to science education, teacher education, structuring theoretical fields, nationality and realism. 


\section{INTRODUCCIÓN}

En la didáctica de las ciencias actual existe un reconocimiento generalizado de que la formación en la filosofía e historia de la ciencia debería ser uno de los componentes fundamentales de la alfabetización científica general de la población (Millar y Osborne, 1998). Hoy en día se concede en el ámbito académico una importancia similar al aprendizaje de los contenidos y procedimientos científicos y al aprendizaje acerca de la propia naturaleza de la ciencia y de su relación con la sociedad y la cultura (Matthews, 1994; Duschl, 1997; McComas et al., 1998). Consecuentemente, los currículos de ciencias de muchos países han comenzado a incorporar recomendaciones y contenidos que apuntan en esta dirección (Matthews, 1998).

Este reconocimiento de la importancia de la filosofía de la ciencia en el aula se traduce naturalmente en la necesidad de introducir los contenidos metacientíficos en el currículo de formación inicial y continuada del profesorado de ciencias. Entre otras muchas cosas, la filosofía de la ciencia ayuda a los profesores a explicitar, comunicar y estructurar sus ideas acerca de la naturaleza de la ciencia (Mellado y Carracedo, 1993) y, consecuentemente, puede derivar en una mejora de su desempeño profesional. En este sentido, podemos afirmar a modo de hipótesis de trabajo que la dimensión metacientífica (filosófica, histórica y sociológica de la ciencia) es una de las parcelas fundamentales del conocimiento profesional del profesorado, capaz de dar estructura y coherencia a las demás (Adúriz-Bravo et al., en prensa).

Ahora bien, diversos diagnósticos (Koulaidis y Ogborn, 1989; Aikenhead y Ryan, 1992; Lederman, 1992; Meyling, 1997; Fernández Montoro, 2000; Gwimbi, 2000; Adúriz-Bravo et al., en prensa) han detectado en el profesorado de ciencias ideas epistemológicas que no se corresponden con las que actualmente sostiene la filosofía de la ciencia. Estas ideas ni siquiera están completamente adecuadas a modelos formales elaborados durante la primera mitad del siglo $\mathrm{xx}$, como puede ser el positivismo lógico. Más bien, las ideas de los profesores acerca de la naturaleza de la ciencia son cercanas a las que se sustentan desde el sentido común, es decir, a aquéllas detentadas por el público no especializado (Pomeroy, 1993). Además, estas ideas se organizan en sistemas de baja coherencia interna que no excluyen ambigüedades y contradicciones (Hodson, 1993). Por último, las ideas acerca de la naturaleza de la ciencia pueden ser inconsistentes con el pensamiento del profesor acerca de la enseñanza y el aprendizaje de las ciencias (Porlán y Rivero, 1998).

En el contexto de estos diagnósticos, McComas (1998) señala quince mitos acerca de la naturaleza de la ciencia que están profundamente arraigados en la práctica del profesorado de ciencias en todo el mundo. Entre ellos, algunos particularmente importantes por sus consecuencias negativas en la imagen de la ciencia que se transmite en la escuela son los que atañen a la universalidad y rigidez del método científico, la objetividad a toda prueba de la ciencia, la validez absoluta del conocimiento científico, el avance de la ciencia por acumulación, el carácter exclusivamente experimental de la ciencia y la posición realista ingenua.

Para atacar estas ideas empobrecidas y construir una visión más compleja de la ciencia que ayude a su enseñanza, resulta necesario seleccionar y transponer algunos contenidos de la filosofía de la ciencia e introducirlos en la formación inicial y continuada del profesorado, relacionándolos con los propios contenidos de ciencias y con los de la didáctica específica. En este trabajo presentamos un esquema teórico para sustentar y organizar la selección de los contenidos metateóricos y lo aplicamos a fin de valorar algunas propuestas de enseñanza de la filosofía de la ciencia para el profesorado generadas por diversos autores y disponibles actualmente en la literatura didáctica. Nuestro esquema combina un análisis curricular clásico de cuatro dimensiones y un análisis de contenidos específicos por medio de lo que hemos dado en llamar criterios teóricos estructurantes.

\section{LA FILOSOFÍA DE LA CIENCIA COMO CONTENIDO A ENSENAR}

Dentro de la didáctica de las ciencias, la enseñanza de la filosofía de la ciencia per se y como auxiliar de las propias ciencias ha ocupado un lugar marginal y, aunque la reflexión teórica sobre esta cuestión está expandiéndose rápidamente en la literatura especializada como una línea de investigación poderosa, aún queda mucho por hacer en lo que atañe a la generación de propuestas prácticas, tanto para el aula de secundaria y bachillerato como para la formación del profesorado (Matthews, 1998).

Un problema central para llevar la filosofía de la ciencia al currículo surge de las diferentes visiones que actualmente coexisten acerca de la naturaleza de la ciencia, algunas de ellas contradictorias entre sí (Stinner, 1992; Jiménez Aleixandre, 1995; Duschl, 1997). La filosofía de la ciencia es una disciplina muy activa cuya producción académica se ha organizado en varias escuelas de investigación en competencia. Se hace necesario, entonces, realizar una selección de contenidos metacientíficos provenientes de estas escuelas que resulte teóricamente coherente y a la vez adecuada a las finalidades generales de la educación científica y a los modelos didácticos disponibles (Izquierdo, 2000). Esta selección más bien pragmática hecha desde la didáctica de las ciencias puede no corresponderse con los desarrollos punteros de la filosofía de la ciencia o bien combinar elementos que provienen de escuelas metacientíficas con bases teóricas encontradas.

McComas y otros (1998) sugieren que disponemos actualmente de una visión más o menos consensuada sobre los contenidos mínimos acerca de la naturaleza de la ciencia que es conveniente incorporar en la educación obligatoria (entre los 5 y los 16 años). Estos autores caracterizan esta visión consensual a través de catorce afirmaciones, que en buena medida provienen de la 
llamada nueva filosofía de la ciencia, desarrollada en la década de los sesenta y representada por los nombres de Thomas Kuhn, Stephen Toulmin e Imre Lakatos. Entre estas afirmaciones destacan, por su importancia central para el aula de ciencias, las que se refieren a la tentatividad del conocimiento científico, la pluralidad metodológica, la carga teórica de la observación, las relaciones entre ciencia y tecnología, y la ciencia como una empresa histórica y socialmente situada, que evoluciona en el tiempo. Varias de estas ideas avanzadas suponen una ruptura con los problemas clásicos de la filosofía de la ciencia positivista lógica (Estany, 1993), cerca de la cual puede ubicarse la epistemología espontánea de profesores y alumnos (Adúriz-Bravo et al., en prensa).

Estas ideas básicas sobre la naturaleza de la ciencia, y algunas más propuestas por otros autores, pueden ser llevadas al currículo de secundaria y de bachillerato y a la formación del profesorado por medio de una serie de actividades diversas, preferiblemente centradas en los contenidos específicos de ciencias (Jiménez Aleixandre, 1995; Izquierdo, 2000), que permiten la reflexión crítica acerca de la ciencia, su desarrollo y su papel en la sociedad, sin exigir el despliegue de modelos teóricos complejos provenientes de la filosofía de la ciencia académica.

Hemos dicho que se han generado diversas propuestas para introducir la reflexión sobre la naturaleza de la ciencia en el ámbito de la formación del profesorado. Estas propuestas están disponibles actualmente en el campo de la didáctica de las ciencias y han sido parcialmente recogidas por algunos autores (McComas, 1998; Abd-el-Khalick y Lederman, 1999; Seroglou y Koumaras, 2001). Nos interesa aquí estudiar esta colección de propuestas mediante una serie de categorías de análisis generales y específicas que nos permitan compararlas y valorarlas. Una valoración teóricamente sustentada permitiría al formador de profesorado seleccionar y adaptar aquellas propuestas que encuentre más pertinentes y mejor adecuadas para su contexto particular de trabajo, y a la vez identificar aquellos puntos en los cuales estas propuestas pueden ser complementadas o mejoradas.

\section{DIMENSIONES GENERALES DE ANÁLISIS PARA ESTUDIAR LAS PROPUESTAS DE ENSEÑANZA}

Las diferentes propuestas que han aparecido durante el siglo xx para enseñar filosofía de la ciencia dentro de la formación científica general pueden ser estudiadas de acuerdo con cuatro dimensiones iniciales que resultan de interés teórico. Estas dimensiones son las poblaciones, los contextos, las finalidades y los formatos de cada propuesta (Fig. 1), y se corresponden respectivamente con las preguntas curriculares clásicas: ¿a quién enseñar la filosofía de la ciencia?, ¿dónde y cuándo enseñarla?, ¿para qué enseñarla? y ¿cómo enseñarla? (dejamos la pregunta ¿qué filosofía de la ciencia enseñar? para las dimensiones relacionadas con el análisis de contenidos, en la siguiente sección). En los apartados que siguen desarrollamos brevemente cada una de estas cuatro dimensiones curriculares generales.

Figura 1

Dimensiones de nuestro marco de análisis de las propuestas de enseñanza de la filosofía de la ciencia.

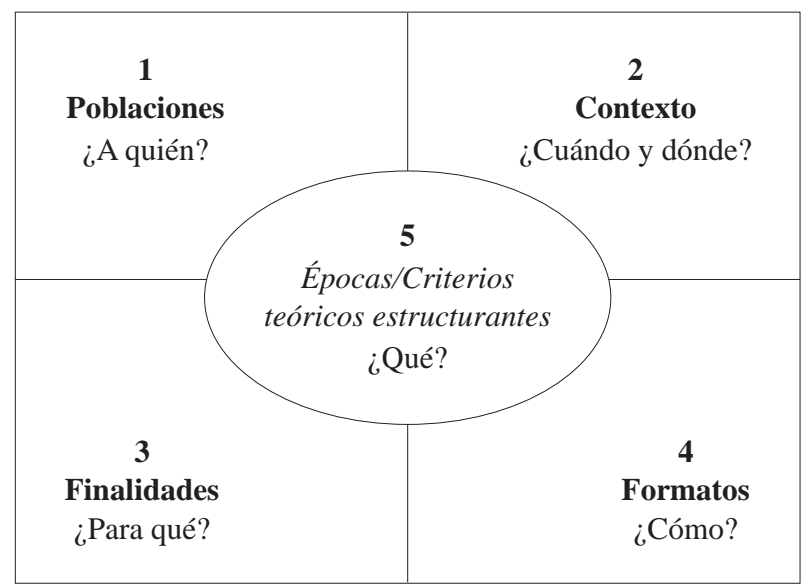

\section{Las poblaciones}

Aunque este trabajo está específicamente centrado en la formación inicial del profesorado de ciencias, existen varias poblaciones de estudiantes que reciben la atención de la didáctica de las ciencias, y a quienes se desea enseñar la filosofía de la ciencia por sus distintos valores. Por una parte, tenemos a los estudiantes de ciencias desde la educación infantil hasta el nivel universitario avanzado. Entre ellos, podríamos distinguir la población general que recibe la escolarización obligatoria (5 a 16 años) y las poblaciones que reciben enseñanza especializada en ciencias en el nivel del bachillerato y la universidad. Una nueva distinción podría ser hecha entre quienes reciben una formación en ciencias complementaria a sus estudios (por ejemplo, ingenieros, médicos) y quienes obtendrán un título en una disciplina científica en particular.

Una segunda población está constituida por el profesorado de ciencias, como se apuntó anteriormente. Esta población se solapa en parte con la primera, ya que en muchos países la formación del profesorado de ciencias para secundaria es equivalente o muy similar a la de los propios científicos. Sin embargo, creemos que es conveniente distinguir ambas poblaciones tanto desde el punto de vista de nuestro análisis teórico como a la hora de seleccionar contenidos específicos de la filosofía de la ciencia. En este sentido, sugerimos que la formación epistemológica del profesorado debería abordar en forma explícita la cuestión instrumental de cómo mejorar la propia práctica utilizando conscientemente algunos modelos de la fílosofía de la ciencia (Izquierdo, 1999b). 
Dentro de la población del profesorado, una distinción clásica que continúa siendo útil es entre el profesorado en formación y el profesorado en activo, ya que estos dos grupos plantean exigencias didácticas diferenciadas. En particular, se ha intentado prestar atención al grupo de los profesores en activo a la hora de implementar propuestas que integren la filosofía de la ciencia con la práctica real de aula (Jiménez Aleixandre, 1995; McComas, 1998).

Una tercera población, por último, sería la de los propios didactas de las ciencias (formadores de profesorado, diseñadores de currículo, escritores de textos, investigadores), con sus características específicas que la diferenciarían en algunos aspectos de la anterior. Parte de esta población de didactas es además la encargada de transponer la filosofía de la ciencia con el fin de contribuir de variadas formas a la educación científica, funcionando así a modo de enlace entre ambas comunidades académicas (Adúriz-Bravo, 1999).

\section{Los contextos}

Centrados específicamente en la población del profesorado de ciencias, McComas y otros (1998) proponen cuatro contextos distintos en los cuales puede introducirse la enseñanza de la filosofía de la ciencia. Uno de ellos es la propia práctica de la ciencia en el ámbito de la formación inicial en las facultades de ciencias. Los otros tres contextos son de mayor interés para nosotros, por lo que los recuperamos aquí en nuestro análisis. Podemos incluir los contenidos metacientíficos en cursos de ciencias, en cursos de didáctica de las ciencias o en cursos especiales de metaciencias (filosofía e historia de la ciencia).

Resulta interesante, y hasta cierto punto necesario, introducir la filosofía de la ciencia para el profesorado en los tres contextos anteriores, complementando y potenciando así su contribución al conocimiento profesional del futuro profesor. Cada uno de los tres contextos tomaría transposiciones específicas del contenido metacientífico adecuadas a distintos objetivos, convergentes entre sí, y relacionadas desde diversos niveles con el contenido científico propiamente dicho. Pero, dadas las limitaciones de tiempo presentes en la formación inicial del profesorado, creemos que el contexto más adecuado para introducir la filosofía de la ciencia es el de la didáctica de las ciencias (Jiménez Aleixandre, 1995; Izquierdo, 1999b). Esta disciplina tendría un mayor poder integrador, permitiendo a la filosofía de la ciencia funcionar como reflexión de segundo orden sobre los propios contenidos científicos, su enseñanza y su aprendizaje (López Rupérez, 1990). Con esta reflexión, a su vez, se podrían generar propuestas didácticas exitosas fundamentadas metacientíficamente (Izquierdo, 1999a; Izquierdo y Adúriz-Bravo, en revisión).

\section{Las finalidades}

La necesidad de la introducción de la filosofía de la ciencia en el currículo general de ciencias en todos los niveles educativos ha sido sustentada in extenso por varios autores (Matthews, 1994; Driver et al., 1996; Duschl, 1997; Izquierdo, 2000). Estos autores han propuesto diversas finalidades para la enseñanza de la filosofía de la ciencia en el aula que pueden ser trasladadas por analogía a la formación del profesorado. Entre estas finalidades, hemos seleccionado por su importancia algunas que mencionaremos brevemente a continuación.

En primer lugar, la filosofía de la ciencia tiene un valor cultural intrínseco análogo al de las propias ciencias. En este sentido, se constituye como un contenido relevante en el currículo de la población general, de los futuros científicos y del profesorado de ciencias como cualquier otra área curricular específica, y debería participar de forma balanceada con respecto al resto de los contenidos científicos. Esta afirmación está avalada por las orientaciones actuales del diseño curricular en ciencias en muchos países, que privilegian la aculturación científica (el conocimiento acerca del papel de la ciencia en la historia de la humanidad) frente a la sola acumulación de contenidos científicos con perfil enciclopedista (Matthews, 1998). La finalidad cultural está además relacionada con objetivos tales como el democrático y el moral (Driver et al., 1996), que son aquéllos en los cuales la filosofía de la ciencia contribuye a la toma de decisiones fundamentadas y críticas acerca del desarrollo científico y tecnológico de las sociedades.

En segundo lugar, la filosofía de la ciencia tiene un valor específico, en tanto que reflexión teórica (esto es, por medio de modelos) acerca de la ciencia. Este valor específico complementa y potencia el de los propios contenidos de ciencias proporcionando una imagen más dinámica y completa, y menos normativa y dogmática, de la empresa científica. Tal imagen significativa es un objetivo deseable para el conjunto general de la población, aquéllos que no serán científicos pero podrán intervenir activamente en decisiones acerca de la ciencia.

Por último, la filosofía de la ciencia tiene un valor instrumental añadido. Diversos autores han mostrado cómo la filosofía de la ciencia puede contribuir a una mejor comprensión de los propios contenidos de ciencias, funcionando como auxiliar en su enseñanza y en su aprendizaje (Driver et al., 1996; Meyling, 1997; Monk y Osborne, 1997; Izquierdo, 1999b), en el desarrollo curricular en ciencias, e incluso en la comprensión y utilización en el aula de modelos didácticos actuales tales como aquéllos de raigambre constructivista (Duschl, 1997).

\section{Los formatos}

Las propuestas de enseñanza de la filosofía de la ciencia que hemos recolectado ofrecen una gran variedad de abordajes didácticos, determinados en parte por los objetivos específicos, el contexto curricular y los modelos de enseñanza y aprendizaje que éstas sustentan. Por formato entendemos este aspecto curricular formal que hace a la naturaleza de las actividades didácticas propuestas y a la forma en que estas están estructuradas y secuenciadas. 
Como formatos en sentido amplio podemos pensar en clases magistrales, talleres, trabajos en pequeños grupos, prácticas de laboratorio, investigaciones guiadas, dramatizaciones y otros. Dentro de estos formatos generales se ubican las distintas actividades específicas que propone cada autor. Las actividades didácticas que seleccionamos y discutimos en este trabajo pueden dar al lector una idea de la amplia variedad existente.

\section{DIMENSIONES ESPECÍFICAS DE ANÁLI- SIS RELACIONADAS CON EL CONTENIDO DE LAS PROPUESTAS: ¿QUE FILOSOFÍA DE LA CIENCIA ENSEÑÁR?}

Considerar la cuestión de qué enseñar lleva a plantearse la necesidad de una serie de criterios teóricos fuertes para la selección de contenidos y tópicos de la filosofía de la ciencia y para la estructuración de estos contenidos en actividades didácticas. También son necesarios criterios de selección de las fuentes utilizadas y de los materiales de apoyo. Dos sencillas dimensiones iniciales que permiten clasificar los contenidos de las propuestas de enseñanza son aquéllas que atienden a:

1) La relación con una disciplina particular. Las propuestas pueden estar ligadas a una disciplina científica en particular (física, química, biología) o ser inespecíficas, aplicables al conjunto de las ciencias naturales.

2) El anclaje en contenidos específicos. Muchos tópicos de la filosofía de la ciencia (como el método hipotéticodeductivo, el debate realismo versus instrumentalismo, o las revoluciones científicas) no están anclados en los contenidos de una disciplina en particular; son aplicables al análisis de cualquier episodio científico. Otros tópicos, en cambio, resultan de ciertas cuestiones más puntuales que se plantean con respecto a conceptos, términos y modelos específicos, como puede ser el caso del análisis de la ontología subyacente a la mecánica cuántica.

A estas dos dimensiones iniciales sumamos nuestra propuesta teórica específica (Fig. 1): qué enseñar se analiza con base en las grandes escuelas de la filosofía de la ciencia que cada propuesta selecciona y a los tópicos particulares, o cuestiones epistemológicas centrales, sobre los cuales la propuesta se concentra. A continuación, presentamos una modelización de la historia de la filosofía de la ciencia del siglo xx en tres épocas y un análisis estructural de sus grandes tópicos alrededor de seis criterios teóricos estructurantes. La combinación de estas dos herramientas con el resto de las dimensiones generales puede proveer una «radiografía» teórica de las diferentes propuestas de enseñanza de la filosofía de la ciencia que contribuya a su aplicación y su adaptación en el ámbito de la formación del profesorado.

\section{Las épocas}

La enseñanza de la filosofía de la ciencia de una manera más o menos formal requiere de una selección explícita de aquellos autores y escuelas que se consideran más pertinentes para la formación del profesorado de ciencias. Aunque la reflexión teórica acerca de la ciencia es tan antigua como la ciencia misma, no es sino hasta inicios del siglo $\mathrm{xx}$ que se constituye como disciplina académica independiente con un perfil epistemológico propio y un cuerpo profesional de investigadores (Estany, 1990, 1993). Por tanto, la conformación del Círculo de Viena en los años veinte, que marca el inicio de la escuela llamada positivismo lógico, puede ser considerada un punto de partida consistente para la selección de los contenidos metacientíficos más adecuados al currículo de formación inicial del profesorado de ciencias.

Partiendo de este hito fundacional, los diversos desarrollos de la filosofía de la ciencia del siglo xx podrían ser periodizados en tres grandes épocas, que desarrollaremos brevemente a continuación. Nuestra periodización (Adúriz-Bravo, 1999) tiene el interés de que las épocas se solapan en el tiempo, manifestando el hecho de que las diferentes escuelas de la filosofía de la ciencia perviven y coexisten, a modo de tradiciones de investigación en competencia conceptual.

\section{El positivismo lógico y la concepción heredada}

Esta primera época abarcaría desde la constitución del Círculo de Viena, a mediados de la década de los veinte, hasta la decadencia de la concepción, heredera de aquél en el ámbito anglosajón, que puede ser señalada con la aparición del libro de Thomas Kuhn, La estructura de las revoluciones científicas, en 1962. Esta época está caracterizada por un énfasis en el aspecto metodológico de las ciencias (Echeverría, 1999) y un enfoque de tipo primordialmente sintáctico (esto es, lógico y lingüístico) para el análisis estructural del conocimiento científico.

Esta primera época de la filosofía de la ciencia tiene gran interés dentro de la didáctica de las ciencias debido a que los modelos generados por el positivismo lógico constituyen una primera formalización de las ideas del sentido común acerca de la naturaleza de la ciencia (Chalmers, 1984). Consecuentemente, sus desarrollos teóricos pueden contribuir a comprender, dentro de la investigación didáctica, las concepciones epistemológicas alternativas del profesorado y servir como puerta de acceso a las cuestiones fundamentales de la filosofía de la ciencia. A esto se suma el hecho de que una comprensión profunda de los desarrollos teóricos de la filosofía de la ciencia posteriores a la segunda guerra mundial tiene como prerrequisito necesario el manejo de ciertas ideas, términos y problemas fundamentales inspeccionados y debatidos con gran detalle por el positivismo lógico y sus seguidores (Matthews, 1998).

\section{El racionalismo crítico y la nueva filosofía de la ciencia}

Esta segunda época abarcaría desde las críticas tempranas que suscita el Círculo de Viena en Francia (Bachelard, 1938) y Alemania (Popper, 1934/1967), hasta la absorción a fines de los años ochenta, por parte de la 
sociología de la ciencia, del enfoque historicista iniciado por Kuhn. Esta época está marcada, inicialmente, por un interés en rebatir o reformar la concepción positivista lógica, llevando el análisis a nuevos campos (externalismo) y planteando cuestiones inexploradas en el acercamiento clásico (Estany, 1993). El mecanismo de apertura propugnado por las corrientes de esta época tiene su pieza clave en el uso de la historia de la ciencia como fuente de escenarios empíricos contra los cuales evaluar los modelos epistemológicos, especialmente los referidos a la dinámica científica (Estany, 1990).

Cabe destacar que la mayor parte de la filosofía de la ciencia que circula actualmente en la didáctica de las ciencias, dirigida tanto a la enseñanza como a la investigación, proviene de esta segunda época (AdúrizBravo, 1999). Los modelos revolucionistas de cambio científico y el falsacionismo de Popper tienen una gran influencia en la investigación didáctica, en el desarrollo curricular de muchos países y en la práctica real del profesorado de ciencias.

\section{El postmodernismo y las visiones contemporáneas}

El auge de las visiones sociologistas en la filosofía de la ciencia, de fuerte corte relativista, puede ser ubicado temporalmente por medio de la aparición del polémico trabajo de Paul Feyerabend (1975), que marcaría el inicio de esta tercera época. Los modelos epistemológicos que agrupamos bajo el nombre general de postmodernismo tienen en común su ataque frontal al concepto de racionalidad categórica, al que buscan alternativas más o menos extremas que desdibujan la especificidad de la ciencia frente a otras formas de conocimiento institucionalizado.

Por otra parte, y desmarcándose de la corriente anterior, las visiones contemporáneas (estructuralista, semántica y cognitiva, entre otras) recuperan cuestiones clave de la filosofía de la ciencia clásica, tales como la relación entre realidad y predicación, o el problema de la reconstrucción racional del conocimiento científico, pero abordándolas desde una perspectiva eminentemente semántica y centrada en el concepto de modelo (ver un resumen en Estany, 1993). Como señala Izquierdo (1999a, 2000), muchos de estos modelos contemporáneos son de una gran riqueza para la didáctica de las ciencias, debido a las profundas relaciones que establecen entre la filosofía de la ciencia, la psicología cognitiva y la pedagogía.

\section{Los criterios teóricos estructurantes}

En este apartado presentamos el núcleo de nuestra propuesta en la organización teórica de los contenidos de la filosofía de la ciencia para la formación del profesorado de ciencias. Hemos denominado a nuestra técnica de análisis composicional criterios teóricos estructurantes. Esta propuesta sigue la tónica general, ya esbozada por varios autores (Koertge, 1990; Estany, 1993; Mellado y Carracedo, 1993; McComas, 1998; Izquierdo, 1999b; Monk y Dillon, 2000), de identificar las ideas clave en la filosofía de la ciencia con el fin de enseñarlas. Sin embargo, nuestro enfoque difiere de los otros en el hecho de que busca dilucidar la estructura que toman estas ideas «soporte» dentro de la disciplina académica.

\section{El concepto de criterio estructurante}

El análisis formal de la estructura de las disciplinas científicas eruditas y escolares ha encontrado en el constructo de idea estructurante una herramienta de suma utilidad. Las ideas estructurantes serían conceptos disciplinares de muy alto nivel de inclusividad y abstracción, capaces de organizar teóricamente los distintos conceptos y modelos presentes en el currículo (Gagliardi, 1986; Sanmartí e Izquierdo, 1997). En este sentido, se trataría de los ejes directores de la organización sintáctica y curricular de un área específica de conocimiento. Para Izquierdo (1999b), incluso, la estructuración dejaría al descubierto los modelos irreductibles de las disciplinas.

Ahora bien, en cualquier disciplina científica más o menos madura, las ideas estructurantes son muy abundantes y aparecen organizadas con coherencia en conjuntos densamente ligados que constituyen áreas temáticas o aspectos de la disciplina. Estos aspectos crecen agrupados en torno a cuestiones clásicas que son las que recorren la disciplina desde su inicio formalizado. Llamamos criterios teóricos estructurantes a los conjuntos coherentes de ideas fundamentales que otorgan identidad a una disciplina académica cuando son utilizados con el fin clasificatorio de discriminar entre propuestas de enseñanza de esa disciplina.

En varios trabajos anteriores (Adúriz-Bravo, 2000; Adúriz-Bravo e Izquierdo, en prensa; Adúriz-Bravo, Izquierdo y Estany, 2001) hemos ido desarrollando un marco de organización de los contenidos de la filosofía de la ciencia y de clasificación de las propuestas de enseñanza según estos criterios estructurantes. En los siguientes apartados caracterizamos brevemente los seis criterios teóricos que hemos construido.

\section{Correspondencia y racionalidad}

Una de las cuestiones más antiguas abordadas por la filosofía de la ciencia es la que se refiere a la naturaleza y alcance de la correspondencia que existe entre el conocimiento científico y la realidad por él representada. Las respuestas clásicas a esta cuestión pueden ser agrupadas en dos grandes posiciones: el realismo, por un lado, y las posturas antirrealistas opuestas a él, entre las cuales destaca, por su gran difusión, el instrumentalis$m o$. La visión realista plantea alguna forma del principio de correspondencia, que establece una relación más o menos biunívoca entre los términos científicos y las entidades del mundo natural. La postura instrumentalista, en cambio, supone que las entidades teóricas son herramientas formales de organización del mundo de fenómenos que no necesariamente tienen contrapartida en la realidad ontológica (Koertge, 1990; Estany, 1993).

El concepto de racionalidad, por otra parte, también ha admitido dos grandes tipos de análisis. El enfoque racio- 
nalista plantea una idea a priori de racionalidad generalmente anclada en la lógica del pensamiento hipotéticodeductivo; esta idea se utiliza luego como criterio de evaluación del desarrollo de las disciplinas científicas. Entre los enfoques antirracionalistas, destaca el que trabaja con el concepto de racionalidad moderada, suponiendo que la empresa científica es racional en tanto que alcanza una alta coherencia instrumental entre medios y fines (Giere, 1992).

\section{Representación y lenguajes}

La concepción epistemológica clásica ha seleccionado la teoría como la unidad de análisis más pertinente para la reconstrucción racional de las ciencias. El estudio exhaustivo de la estructura de las teorías desde el punto de vista lógico y lingüístico ha sido el gran proyecto intelectual de los filósofos de la ciencia de la primera mitad del siglo xx. Esta concepción basada en teorías (Giere, 1992) ha privilegiado el lenguaje formal o especializado, entendido como un perfeccionamiento del lenguaje natural, como la forma de representación científica por excelencia (Hempel, 1988).

En las últimas décadas ha emergido y se ha consolidado un campo interdisciplinar de estudios conocido como ciencia cognitiva, extensamente aplicado al estudio de la ciencia y de su enseñanza (Nersessian, 1992; Izquierdo, 1999a). El interés central de esta visión teórica es la consideración de los diversos mecanismos de representación del mundo (particularmente, los modelos) de los que dispone el ser humano. En la filosofía de la ciencia también se ha dado este giro cognitivo con la aparición de la llamada concepción basada en modelos (model based view) para explicar la estructura y evolución de las ciencias eruditas (Giere, 1992). Dicha concepción se ha transferido a la didáctica de las ciencias y está dando lugar a diversas derivaciones (Izquierdo, 1999a, 2000; Izquierdo y Adúriz-Bravo, en revisión).

Ahora bien, tanto las teorías como los modelos, en tanto que representaciones, se expresan y se comunican por medio de diversos lenguajes (lenguaje científico formal, lenguaje simbólico, lenguaje computacional, fórmulas, gráficas, analogías). La concepción clásica analizaba el lenguaje científico escrito en términos de sus conceptos constituyentes y de las relaciones entre ellos. Las visiones actuales, desde una perspectiva más cercana a las de la pragmática y la retórica, recuperan el valor de la abducción, la analogía y la metáfora como mecanismos de construcción de sentido en la ciencia (Izquierdo, 1999b; Osborne, 1999), a la vez que propugnan por una concepción multisemiótica del lenguaje científico, que incluya de manera coordinada las diversas formas de representación.

\section{Intervención y método}

El método científico ha sido desde antiguo una preocupación central de los filósofos de la ciencia, hasta tal punto que los modelos metodológicos han constituido durante buena parte del siglo xx el componente principal de la disciplina (Echeverría, 1999). Alrededor de esta temática se han dado las más variadas posiciones. Hay quienes consideran que existe un único método científico, entendido como una secuencia rígida de pasos para generar o validar conocimiento. Frente a esta visión de corte neopositivista, se han propuesto diversas variantes más o menos «irracionales», que van desde la pluralidad metodológica (en la visión secesionista: Estany, 1993) hasta la ausencia total de método científico establecido, en la corriente postmodernista.

Los debates alrededor de la metodología científica ponen en el centro de atención de la filosofía de la ciencia la cuestión de la intervención (representacional, discursiva y material) que la ciencia hace sobre el mundo. El acercamiento clásico a esta cuestión separó tajantemente las dimensiones teórica y experimental de la ciencia. Algunas de las visiones actuales más sugerentes, por ejemplo, Hacking (1983), rescatan el componente de transformación activo del mundo que tienen los propios modelos teóricos y proporcionan una visión sistémica de la ciencia en la cual interactúan objetivos, teorías y métodos.

\section{Contextos y valores}

Una distinción tradicional en la filosofía de la ciencia es la que se estableció tempranamente entre el contexto de descubrimiento y el contexto de justificación (Reichenbach, 1938). La filosofía se centró en este último intentando dilucidar los mecanismos lógicos por los cuales el conocimiento científico es validado e ingresa al cuerpo de la ciencia establecida. La distinción, además, sirvió para desdibujar un tanto los problemas de evolución histórica de la ciencia (Estany, 1990). La irrupción del externalismo y la historia en la filosofía de la ciencia obligó en cierta manera a repensar esta dicotomía y a buscar visiones de síntesis que articularan el plano cognitivo y el plano social (Giere, 1992).

Otro contexto de importancia innegable para comprender la naturaleza de la ciencia es el contexto de aplicación, que funciona a modo de vínculo entre la ciencia y la tecnología, permitiendo un estudio más rico e integrado de ambas. Javier Echeverría (1995) añade además, a estos contextos, un cuarto, el de educación o difusión, que alcanza una importancia central en el propio desarrollo conceptual de la ciencia a lo largo de su historia (Izquierdo, 1999b, 2000).

\section{Evolución y juicio}

En sus inicios, la filosofía de la ciencia académica estuvo poco preocupada por el estudio de la evolución de las disciplinas científicas y el problema del cambio conceptual (Estany, 1990). Se suponía tácitamente que la fuerte visión sincrónica construida por el positivismo lógico podía extenderse en el tiempo por medio de cierto principio de avance por acumulación. El trabajo de Thomas Kuhn cuestionó profundamente este enfoque sincrónico introduciendo la hipótesis de que, en determinados períodos de la ciencia, se podían observar evoluciones anómalas y alineales, asimilables a verdaderos cambios de Gestalt. 
El estudio de la dinámica científica trajo consigo la atención a la naturaleza del juicio científico, es decir, a los mecanismos por los cuales los científicos individuales y la comunidad en su conjunto seleccionan los modelos teóricos con los cuales consideran válido representarse el mundo. Algunos modelos actuales de juicio científico plantean una imagen evolucionista, que involucra la selección de las estrategias cognitivas en el bagaje cultural humano (Giere, 1992, 1999).

\section{Normatividad y recursión}

Este último criterio se refiere a la reflexión que la propia filosofía de la ciencia hace sobre su rol como metaciencia y su papel en el conjunto de las ciencias. En tanto que disciplina de segundo orden, o metadiscursiva (Koertge, 1990; Estany, 1993; Adúriz-Bravo, 1999), la filosofía de la ciencia plantea modelos teóricos acerca de las ciencias que a su vez pueden servir para intervenir sobre ellas y guiar su desarrollo.

En este sentido, una discusión que actualmente está en su apogeo es la que se refiere a la naturaleza de los saberes metacientíficos. Se reconocen dos posturas principales en torno a esta cuestión: la normatividad y el naturalismo (entendidos ambos en sus versiones extremas e incompatibles, ya que también hay un naturalismo normativo: Laudan, 1998). El enfoque normativo pretende hallar en la filosofía de la ciencia criterios racionales para determinar la cientificidad del conocimiento y dirigir el avance de las ciencias. La postura naturalista propugna por un abordaje explicativo y acerca metodológicamente la filosofía de la ciencia a las demás disciplinas empíricas.

\section{PROPUESTAS DE ENSEÑANZA DE LA FILOSOFÍA DE LA CIENCIA PARA EL PROFESORADO DE CIENCIAS}

Actualmente existe en diversos países (España, Francia, Reino Unido, Holanda, Dinamarca, Noruega, varios en Latinoamérica) un movimiento de reforma curricular de los estudios iniciales del profesorado de ciencias que propugna por la introducción de un fuerte componente metacientífico (Matthews, 1998). Paralelamente a este movimiento, asistimos a una intensa producción académica ocupada de estudiar la incorporación de la filosofía de la ciencia en la didáctica de las ciencias y en la educación científica. Ahora bien, la necesidad urgente de esta incorporación está generando un volumen de trabajos específicos que va en rápido crecimiento. Conforme la filosofía de la ciencia y la didáctica de las ciencias se integran más profundamente, las propuestas prácticas para enseñar la filosofía de la ciencia se hacen más abundantes y más fácilmente asequibles para profesores y didactas.

El propósito de esta sección es utilizar los criterios teóricos estructurantes como organizadores para recoger y analizar algunas propuestas de enseñanza de la filosofía de la ciencia que circulan en la literatura espe- cializada de la didáctica de las ciencias. De nuestro análisis, el lector podrá inferir cómo el marco teórico que presentamos puede ser útil a la hora de adaptar propuestas didácticas a sus necesidades particulares. Por razones de concisión, trataremos brevemente seis ejemplos, cada uno de los cuales se enfoca prioritariamente en uno de los criterios. Para más información puede consultarse Adúriz-Bravo (2000).

\section{El razonamiento científico y las novelas policiales}

El primer ejemplo desarrolla varios aspectos coordinados del campo de correspondencia y racionalidad, principalmente el concepto de razonamiento científico (Gaeta et al., 1996; Giere, 1999). Se trata de una propuesta que introduce tópicos de lógica formal en la formación inicial del profesorado de física, química y biología (Adúriz-Bravo, en prensa). A través de estos tópicos, la propuesta refleja la oposición entre las concepciones sintáctica y semántica de los esquemas de razonamiento científico, enfrentando así modelos provenientes de la primera y la tercera épocas de la filosofía de la ciencia.

Se trabaja con la conocida novela policíaca Muerte en el Nilo de Agatha Christie, y opcionalmente con su versión cinematográfica. Se presenta el llamado esquema de razonamiento abductivo (Samaja, 1994) y con él se examina el proceso que sigue el detective Hercule Poirot en su investigación del crimen. Este proceso se compara luego a la modelización teórica en ciencias; por medio de esta analogía se desarrollan los elementos centrales del contexto de justificación en el modelo inductivodeductivo, o método aristotélico, y en el modelo decisional de Giere (1999).

Las actividades de lápiz y papel que este ejemplo utiliza introducen en el aula la cuestión de la correspondencia a través del análisis del realismo pragmático (Giere, 1992), entendido como relación de similaridad entre un modelo teórico y el mundo. La racionalidad, a su vez, es revisada por medio de la discusión acerca de la pertinencia del uso de esquemas lógicos fuertes (deductivos) para modelizar el pensamiento de los científicos; con ello se reproduce la evolución de la filosofía de la ciencia desde el modelo de explicación de Hempel-Popper (racionalista categórico) hasta la introducción de la racionalidad ampliada en los años sesenta (Gaeta et al., 1996).

\section{La reconstrucción racional de las estructuras teóricas}

El segundo ejemplo proviene del contexto de la formación de licenciados en filosofía (Estany y Casacuberta, 2000) e introduce el campo de representación y lenguajes. Se trata de una serie de ejercicios de lápiz y papel que proponen la reconstrucción formal de diversas estructuras teóricas (provenientes de varias disciplinas científicas naturales y sociales); para ello se utilizan las herramientas conceptuales de los métodos axiomático, conjuntista y semántico. La actividad puede ser complementada con el uso de otros artefactos gráficos para la organización teórica, tales como los mapas de Thagard o los diagra- 
mas de Toulmin (Izquierdo, 2000), que muestran la jerarquía y evolución conceptual respectivamente.

La idea de representación aparece contemplada al considerarse las ventajas y limitaciones del uso de la teoría como unidad funcional y estructural del análisis epistemológico (Adúriz-Bravo, 1999). Por otra parte, la actividad permite un primer acercamiento a una concepción multisemiótica del lenguaje científico, según la cual la definición de los modelos teóricos (Giere, 1992) se realiza tanto a través de las estrategias proposicionales clásicas como del uso metafórico de diversos registros de expresión.

\section{El péndulo y la determinación del patrón de longitud}

El tercer ejemplo ha sido desarrollado por Michael Matthews (2000), y se enfoca principalmente en el campo de intervención y método. Dentro de un trabajo más amplio, el autor explora con detalle varios episodios de la historia de la ciencia que tienen que ver con la formulación del modelo de péndulo en física y la utilización del péndulo como artefacto en la horología (construcción de relojes), la navegación y la estandarización de pesos y medidas.

La propuesta que hemos seleccionado se concentra en la polémica alrededor de la determinación del metro universal por métodos geodésicos o cronométricos, entre los siglos XVII y XVIII. El autor ilumina la oposición que se da entre la sugerencia de Huygens de usar como patrón el péndulo de segundos y la decisión de la Académie francesa de ciencias de adoptar una partición del cuadrante meridiano terrestre.

La idea de intervención aparece tratada al explorarse las profundas transformaciones tecnológicas que introduce el péndulo, hasta ahora descuidadas por los currículos de ciencias. Por otra parte, el método se examina críticamente desde el punto de vista de los factores no epistémicos (en este ejemplo, fundamentalmente políticos) que interfieren con la lógica interna de una investigación científica.

\section{Los cómics y la imagen de ciencia}

El cuarto ejemplo utiliza cómics de divulgación científica y de ciencia ficción (Gallego, 1999) para intentar un cambio en las ideas del sentido común que estudiantes y profesores mantienen acerca de la naturaleza de la ciencia. La autora define una serie de imágenes folk acerca de la ciencia que están difundidas y reforzadas por los medios de comunicación: la individualista, la aproblemática y la descontextualizada, entre otras.

La idea de los diferentes contextos de la actividad científica subyace a la revisión que se hace de las circunstancias particulares en las que se produce un hallazgo; ejemplos tratados en los cómics incluyen los descubrimientos del radio y de la penicilina. Además, la propuesta sugiere un examen crítico de los valores que subyacen a los diferentes estereotipos de los científicos y su trabajo. Particularmente interesante es la llamada de atención sobre la desvalorización que se hace del papel de la mujer en la imagen popular de ciencia.

\section{Diálogo entre un farmacéutico tradicional y un iatroquímico}

El quinto ejemplo (Izquierdo, 2000) se enfoca sobre el campo de evolución y juicio. La confrontación entre dos científicos imaginarios, escrita bajo la forma filosófica clásica de diálogo (Koertge, 1990), sirve a la autora para oponer dos paradigmas de la farmacia en los inicios de la química científica: la herboristería y la iatroquímica. Esta oposición permite confrontar dos visiones del mundo y de la ciencia radicalmente diferentes. La propuesta reconstruye con gran detalle el contexto sociocultural de la ciudad de París en el siglo XVII.

La idea de evolución se explora al evaluar las mejoras que un paradigma supone frente al otro y la nueva visión de ciencia que se propugna. Los elementos que coadyuvan al juicio científico aparecen sugeridos con detalle a lo largo del diálogo. Varias veces, los interlocutores descalifican abiertamente una u otra visión de la farmacia por factores de contexto que no formarían parte de la lógica interna del modelo (por ejemplo, la concepción de la relación entre hombre y naturaleza, las conexiones con la alquimia o incluso el sabor de los medicamentos).

\section{El estatus de la didáctica de las ciencias}

Este último ejemplo (Bonan, 1999) se concentra en el campo de normatividad y recursión. En el contexto de un curso de didáctica de las ciencias para futuros profesores de ciencias, se reflexiona sobre el estatus epistemológico de esta disciplina y sus relaciones con otras disciplinas académicas y con la propia enseñanza de las ciencias.

El componente normativo del conocimiento filosófico se discute al revisar el debate acerca de la cientificidad de la didáctica; los autores que la niegan esgrimen un modelo de ciencia construido a priori sobre la base de las disciplinas más maduras. Por otra parte, el elemento de recursión aparece explícitamente cuando se distinguen los diversos niveles de discurso de las ciencias y su didáctica, y se promueve una reflexión de segundo orden sobre esta disciplina (Adúriz-Bravo, 1999).

\section{COMENTARIOS FINALES}

Los seis criterios teóricos estructurantes que hemos presentado, combinados con nuestra descripción de las épocas y las otras dimensiones, proporcionan un mapa de la filosofía de la ciencia del siglo xx que permite ubicar las diferentes propuestas de enseñanza. En este sentido, se constituyen en una herramienta de análisis curricular. Pero además posibilitan identificar las carencias de cada propuesta, su grado de completitud y de 
coherencia teórica, y la relación con sus fuentes eruditas; por tanto, son también una herramienta de evaluación de la calidad didáctica. Son, por último, una herramienta de diseño de unidades didácticas, pues permiten combinar, modificar y complementar temáticamente las propuestas, sugiriendo nuevas actividades para el aula enfocadas en cada uno de ellos.

Nuestra estrategia de usar las grandes ideas fundacionales de la filosofía de la ciencia con el fin de enseñarla se ha valido de propuestas anteriores que apuntan en la misma dirección; en este aspecto, nos ha sido de gran utilidad revisar libros de texto de filosofía de la ciencia dirigidos a la formación de científicos, filósofos y profesores de ciencias. Por otra parte, el discriminar valorativamente entre modelos teóricos por medio de criterios estructurantes provenientes de la filosofía de la ciencia tiene antecedentes fuertes en el campo de la psicología (Estany, 1999).

Las propuestas de enseñanza de la filosofía de la ciencia que hemos seleccionado aquí y las que hemos relevado en un estudio más extenso (Adúriz-Bravo, 2000) muestran una variedad muy grande en cuanto a los tópicos, tratamientos y enfoques didácticos. Creemos que el aparato conceptual aquí presentado, que combina el uso de las distintas dimensiones de análisis y la técnica de los campos estructurantes, puede resultar una herramienta útil para la selección y adaptación de estas propuestas. En este sentido, esta herramienta puede ser complementada con otros instrumentos de valoración de las propuestas de enseñanza de la filosofía e historia de la ciencia, como los de Abd-el-Khalick y Lederman (1999) y Serouglou y Koumaras (2001).

\section{REFERENCIAS BIBLIOGRÁFICAS}

ABD-EL-KHALICK, F. y LEDERMAN, N. (1999). Success of the attempts to improve science teachers' conceptions of the nature of science: A review of the literature, en Bevilacqua, F. y Giannetto, E. (eds.). Bicentenary of the invention of the battery, 52. Pavia: European Physical Society.

ADÚRIZ-BRAVO, A. (1999). «Elementos de teoría y de campo para la construcción de un análisis epistemológico de la didáctica de las ciencias». Tesis de maestría. Universitat Autònoma de Barcelona.

ADÚRIZ-BRAVO, A. (2000). A theoretical characterisation of proposals integrating the philosophy of science and science education, en Actas de la VI Escuela de Verano de la ESERA, s/pp. Copenhague: The Danish University of Education.

ADÚRIZ-BRAVO, A. (en prensa). Aprender sobre el pensamiento científico en el aula: una propuesta para usar novelas policiales. Alambique.

ADÚRIZ-BRAVO, A. e IZQUIERDO, M. (en prensa). «Structuring» ideas from the philosophy of science for physics teacher education, en Pintó, R. y Suriñach, S. (eds.). International Conference Physics Teacher Education Beyond 2000. Selected contributions. París: Elsevier.

ADÚRIZ-BRAVO, A., IZQUIERDO, M. y ESTANY, A. (2001). A characterisation of practical proposals to teach the philosophy of science to prospective science teachers, en Valanides, N. (ed.). Science and technology education: Preparing future citizens, I, pp. 37-47. Paralimni: University of Cyprus.

ADÚRIZ-BRAVO, A., SALAZAR, I., BADILLO, E., MENA, N., TAMAYO, O., TRUJILLO, J. y ESPINET, M. (en prensa). Ideas on the nature of science in prospective teachers for early childhood education, en Selected Proceedings of the $25^{\text {th }}$ ATEE Annual Conference.

AIKENHEAD, G. y RYAN, A. (1992). The development of a new instrument: Views on Science-Technology-Society. Science Education, 76(4), pp. 477-491.

BACHELARD, G. (1938). La formation de l'esprit scientifique. París: Vrin.

BONAN, L. (1999). Epistemología de la didáctica de las ciencias. Buenos Aires: Universidad de Buenos Aires.

CHALMERS, A. (1984). ¿Qué es esa cosa llamada ciencia? Madrid: Siglo XXI. (2a. ed. original en inglés, 1982).

DRIVER, R., LEACH, J., MILLAR, R. y SCOTT, P. (1996). Young people's images of science. Bristol: Open University Press.

DUSCHL, R. (1997). Renovar la enseñanza de las ciencias. Importancia de las teorías y su desarrollo. Madrid: Narcea. (Ed. original en inglés, 1990).

ECHEVERRÍA, J. (1995). Filosofía de la ciencia. Madrid: Akal.

ECHEVERRÍA, J. (1999). Introducción a la metodología de la ciencia. Madrid: Cátedra.

ESTANY, A. (1990). Modelos de cambio científico. Barcelona: Crítica.

ESTANY, A. (1993). Introducción a la filosofía de la ciencia. Barcelona: Crítica. 
ESTANY, A. (1999). Vida, muertey resurrección de la conciencia. Análisis filosófico de las revoluciones científicas en la psicología contemporánea. Barcelona: Paidós.

ESTANY,A. y CASACUBERTA, D. (2000). Manual de prácticas de filosofía de la ciencia. Barcelona: Crítica.

FERNÁNDEZ MONTORO, I. (2000). «La transformación de las concepciones docentes espontáneas sobre la ciencia». Tesis doctoral. Universitat de València.

FEYERABEND, P. (1975). Contra el método. Barcelona: Ariel. (Ed. original en inglés, 1970).

GAETA, R., GENTILE, N., LUCERO, S. y ROBLES, N. (1996). Modelos de explicación científica. Problemas epistemológicos de las ciencias naturales y sociales. Buenos Aires: Eudeba.

GAGLIARDI, R. (1986). Los conceptos estructurantes en el aprendizaje por investigación. Enseñanza de las Ciencias, 4(1), pp. 30-36

GALLEGO, P. (1999). «El uso del cómic en la imagen de la ciencia». Tesis de maestría. Universitat de València.

GIERE, R. (1992). La explicación de la ciencia. Un acercamiento cognoscitivo. México: Consejo Nacional de Ciencia y Tecnología. (Ed. original en inglés, 1988).

GIERE, R. (1999). Un nuevo marco para enseñar el razonamiento científico. Enseñanza de las Ciencias, núm. extra, pp. 63-70.

GWIMBI, E. (2000). «Relationship between science teachers» philosophies of science and their classroom practices: A pilot survey». Tesis de posgrado. King's College de Londres.

HACKING, I. (1983). Representing and intervening. Cambridge: Cambridge University Press.

HEMPEL, C. (1988). Fundamentos de la formación de conceptos en ciencia empírica. Madrid: Alianza. (Ed. original en inglés, 1952).

HODSON, D. (1993). Philosophy stance of secondary school science teachers, curriculum experiences and children's understanding of science: Some preliminary findings. Interchange, 24(1-2), pp. 41-52.

IZQUIERDO, M. (ed.) (1999a). Aportación de un modelo cognitivo de ciencia a la enseñanza de las ciencias. Enseñanza de las Ciencias, núm. extra.

IZQUIERDO, M. (1999b). Memoria de acceso a la plaza de catedrática. Universitat Autònoma de Barcelona.

IZQUIERDO, M. (2000). Fundamentos epistemológicos, en Perales, F.J. y Cañal, P. (comps.). Didáctica de las ciencias experimentales. Teoría y práctica de la enseñanza de las ciencias, pp. 35-64. Alcoy: Marfil.

IZQUIERDO, M. y ADÚRIZ-BRAVO, A. (en revisión). Epistemological foundations of school science. Science \& Education.

JIMÉNEZ ALEIXANDRE, M.P. (1995). Comparando teorías. La reflexión sobre la naturaleza de la ciencia en la formación del profesorado, en Blanco, L. y Mellado, V. (coords.). La formación del profesorado de ciencias y matemáticas en
España y Portugal, pp. 267-272. Badajoz: Imprenta de la Diputación Provincial.

KOERTGE, N. (1990). Curs de filosofia de la ciència. Barcelona: Edicions de la Magrana. (Ed. en inglés, 1989).

KOULAIDIS, V. y OGBORN, J. (1989). Science teachers' philosophical assumptions: How well do we understand them? International Journal of Science Education, 17, pp. 273-283.

LAUDAN, L. (1998). Naturalismo normativo y el progreso de la filosofía, en González, W. (ed.). El pensamiento de L. Laudan. Relaciones entre historia de la ciencia y filosofía de la ciencia. A Coruña: Universidade da Coruña.

LEDERMAN, N. (1992). Students' and teachers' conceptions of the nature of science: A review of the research. Journal of Research in Science Teaching, 29(4), pp. 331-359.

LÓPEZ RUPÉREZ, F. (1990). Epistemología y didáctica de las ciencias. Un análisis de segundo orden. Enseñanza de las Ciencias, 8(1), pp. 65-74.

MATTHEWS, M. (1994). Science teaching. The role of history and philosophy of science. Nueva York: Routledge.

MATTHEWS, M. (1998). Foreword and introduction, en McComas, W. (ed.). The nature of science in science education. Rationales and strategies, XI-XXI. Dordrecht: Kluwer.

MATTHEWS, M. (2000). Time for science education. Nueva York: Plenum Publishers.

McCOMAS, W. (1998). The principal elements of the nature of science: Dispelling the myths, en McComas, W. (ed.). The nature of science in science education. Rationales and strategies, pp. 53-70. Dordrecht: Kluwer.

McCOMAS, W., CLOUGH, M. y ALMAZROA, H. (1998). The role and character of the nature of science in science education, en McComas, W. (ed.). The nature of science in science education. Rationales and strategies, pp. 3-39. Dordrecht: Kluwer.

MELLADO, V. y CARRACEDO, D. (1993). Contribuciones de la filosofía de la ciencia a la didáctica de las ciencias. Enseñanza de las Ciencias, 11(3), pp. 331-339.

MEYLING, H. (1997). How to change students' conceptions of the epistemology of science. Science \& Education, 6, pp. 397-416.

MILLAR, R. y OSBORNE, J. (eds.) (1998). Beyond 2000. Science education for the future. Londres: King's College.

MONK, M. y DILLON, J. (2000). The nature of scientific knowledge, en Monk, M. y Osborne, J. (comps.). Good practice in science teaching. What research has to say, pp. 72-87. Buckingham: Open University Press.

MONK, M. y OSBORNE, J. (1997). Placing the history and philosophy of science on the curriculum: A model for the development of pedagogy. Science Education, 81(4), pp. 405-424.

NERSESSIAN, N. (1992). How do scientists think? Capturing the dynamics of conceptual change in science, en Giere, $\mathrm{R}$. (ed.). Cognitive models of science, pp. 3-44. Minneapolis: University of Minnesota Press.

OSBORNE, J. (1999). Promoting scientific argument in the classroom: A rhetorical perspective, en Proceedings of the International ESERA Conference, s/pp. Kiel: IPN. 
POMEROY, D. (1993). Implications of teachers' beliefs about the nature of science: Comparison of the beliefs of scientists, secondary science teachers, and elementary teachers. Science Education, 77(3), pp. 261-278.

POPPER, K. (1967). La lógica de la investigación científica. Madrid: Tecnos. (Ed. original en alemán, 1934).

PORLÁN, R. y RIVERO, A. (1998). El conocimiento de los profesores. Sevilla: Díada.

REICHENBACH,H.(1938). Experience and prediction. Chicago: University of Chicago Press.
SAMAJA, J. (1994). Epistemología y metodología. Elementos para una teoría de la investigación científica. Buenos Aires: Eudeba.

SANMARTÍ, N. e IZQUIERDO, M. (1997). Reflexiones en torno a un modelo de ciencia escolar. Investigación en la Escuela, 32, pp. 51-62.

SEROGLOU, F. y KOUMARAS, P. (2001). The contribution of the history of physics in physics education: A review. Science \& Education, 10(1-2), pp. 153-172.

STINNER, A. (1992). Science textbooks and science teaching: From logics to evidence. Science Education, 76(1), pp. 1-16.

[Artículo recibido en marzo de 2001 y aceptado en agosto de 2001.] 